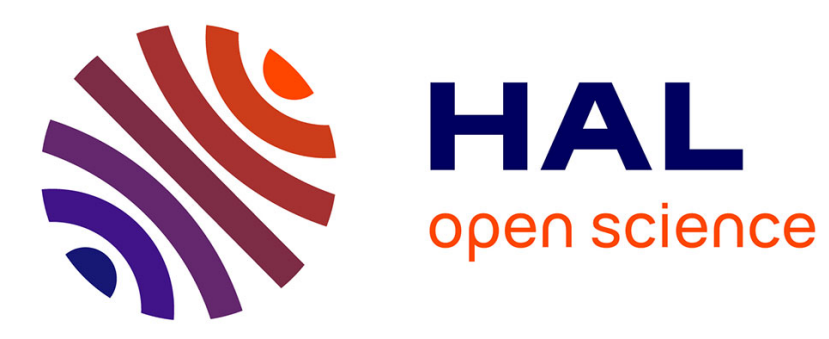

\title{
On the melting of incommensurate structures
}

\author{
I.F. Lyuksyutov, V.L. Pokrovsky
}

\section{To cite this version:}

I.F. Lyuksyutov, V.L. Pokrovsky. On the melting of incommensurate structures. Journal de Physique Lettres, 1982, 43 (1), pp.11-13. 10.1051/jphyslet:0198200430101100 . jpa-00231998

\section{HAL Id: jpa-00231998 https://hal.science/jpa-00231998}

Submitted on 1 Jan 1982

HAL is a multi-disciplinary open access archive for the deposit and dissemination of scientific research documents, whether they are published or not. The documents may come from teaching and research institutions in France or abroad, or from public or private research centers.
L'archive ouverte pluridisciplinaire HAL, est destinée au dépôt et à la diffusion de documents scientifiques de niveau recherche, publiés ou non, émanant des établissements d'enseignement et de recherche français ou étrangers, des laboratoires publics ou privés. 
Classification

Physics Abstracts

$61.12-68.20$

\title{
On the melting of incommensurate structures
}

\author{
I. F. Lyuksyutov (*) and V. L. Pokrovsky \\ L. D. Landau Institute for Theoretical Physics, Chernogolovka 142432, U.S.S.R.
}

(Reçu le 29 juin 1981, accepté le 3 novembre 1981)

\begin{abstract}
Résumé. - Nous calculons la courbe de fusion d'une superstructure unidimensionnelle à solitons dans un cristal incommensurable. Nous montrons que les interactions mécaniques et celles dues à l'entropie sont du même ordre de grandeur sur la courbe de fusion. Nous analysons l'influence des contraintes élastiques dues au substrat.
\end{abstract}

\begin{abstract}
The melting curve of a one-dimensional soliton superstructure in an incommensurate crystal is calculated. Entropic and mechanical interactions are shown to be of the same order of magnitude with respect to their influence on the melting curve. The influence of the elastic strains of the substrate on the phase diagram is analysed.
\end{abstract}

A commensurate crystal arising in a submonolayer of atoms adsorbed onto an anisotropic crystalline substrate has been shown to be unstable under certain conditions [1-3], with respect to the formation of infinitely long defects - solitons. The interaction between the soliton lines results in the formation of an incommensurate crystal, i.e., a crystal of soliton lines. In the work by Lyuksyutov [4] and Coppersmith et al. [5] the dislocation mediated melting of a soliton superstructure in an incommensurate crystal was investigated. In particular, such melting was shown to be possible at an arbitrarily low temperature, provided the soliton lattice constant is sufficiently large. In reference [4] only the exponentially small elastic repulsion between solitons was considered. In this approximation the melting of a soliton lattice proceeds for any type of commensurability. In reference [5] only the repulsion due to the loss of entropy was considered. The $(p \times l)$ type structures were considered as initially commensurate crystals. In the approximation, adopted by the authors of reference [5], soliton structures appeared to be unstable with respect to the creation of single dislocations at $p=1,2$. At larger values of $p$ creation of a single dislocation is thermodynamically unfavourable at any temperature. In the present work both types of repulsion are shown to be of similar importance for the melting curve. Their simultaneous consideration makes it possible to obtain the equation of the melting curve.

We start with the expression for the energy density of a soliton system when the average distance $l \equiv 1 / n$ between solitons for exceeds their width $l_{0}([3], \S 11)$ :

$$
E=\varepsilon n+A \varepsilon_{0} n \exp \left(-1 / l_{0} n\right)+\frac{T^{2} \pi^{2} n^{3}}{6 \varepsilon_{0}} .
$$

(*) Permanent address : Institute of Physics, Ukrainian Academy of Science, Kiev, U.S.S.R. 
Here $\varepsilon=\varepsilon_{0}-\tilde{\lambda} a \delta$ is the energy of the unit length of a soliton line, tending to zero at the point $\delta=\delta_{\mathrm{c}}$ of the commensurate-incommensurate phase transition; $\delta$ is the " natural misfit " which is a linear function of the chemical potential of adatoms in the gaseous phase; $\tilde{\lambda}$ is some combination of elastic constants of an adatom lattice; $a$ is the lattice constant, $A$ is the numerical constant depending on the model under consideration. The compression modulus $K_{1}$ of the soliton lines can be obtained from expression (1) for the energy :

$$
K_{1}=n^{2} \frac{\partial^{2} E}{\partial n^{2}}=\frac{A \varepsilon_{0} \exp \left(-1 / l_{0} n\right)}{l_{0}^{2} n}+\frac{\pi^{2} T^{2} n^{3}}{\varepsilon_{0}}
$$

$\varepsilon_{0}$ is the energy of the unit length of a soliton line at $\delta=0$. According to the theory [1, 2], the same value enters the temperature-dependent energy term, determining the effective mass of a soliton at $T=0$.

The shear modulus $K_{2}$ can be easily associated with the tension of a soliton line considered as a string :

$$
K_{2}=\varepsilon_{0} n \text {. }
$$

The elastic energy $U$ of a soliton lattice can be written as follows :

$$
U=\frac{1}{2} \int \mathrm{d} x \mathrm{~d} y\left[K_{1}\left(\frac{\partial u}{\partial x}\right)^{2}+K_{2}\left(\frac{\partial u}{\partial y}\right)^{2}\right]
$$

where $u$ is the transverse displacement of a soliton line. After a simple change of variables the energy $U$ can be reduced to the Hamiltonian of the $x-y$ model with $K_{1}=K_{2}$. It is possible to use directly the equation for the transition temperature of the Kosterlitz-Thouless theory [6] :

$$
T_{\mathrm{m}}=\frac{\sqrt{K_{1} K_{2}}}{8 \pi} b^{2},
$$

where $b$ is the Burgers vector of dislocation. In the case under consideration in the $(p \times l)$ structures $b=l_{p}=p / n$. Inserting (2) and (3) into (5) we obtain the equation for $T_{\mathrm{m}}$ with the following solution :

$$
T_{\mathrm{m}}=\frac{\varepsilon_{0} \exp \left(-1 / 2 l_{0} n\right)}{\pi^{2} l_{0} n} \frac{A^{1 / 2}}{\left(\frac{64}{p^{4}}-1\right)^{1 / 2}}
$$

Equation (6) shows that the dislocation mediated melting is possible for $p=1,2$ only. The elastic repulsion of solitons increases the energy of a dislocation. As a consequence, the region of stability of an incommensurate structure appears at $p=1,2$. For $p=3$ single dislocations are thermodynamically unfavourable at any temperature. For estimation of the equilibrium density of solitons on the melting curve the term in (1) corresponding to the entropy-mediated repulsion is not important. So, there is a classical relationship between the soliton density $n$ and the value $\delta-\delta_{\text {c }}$ such that (see, e.g. [2])

$$
n \sim\left[l_{0} \ln \left(\delta-\delta_{\mathrm{c}}\right)\right]^{-1} .
$$

The equation of the melting curve in the $(T, \delta)$ plane has the form :

$$
\delta_{\mathrm{m}}-\delta_{\mathrm{c}}=\left(\frac{T}{\varepsilon_{0} l_{0}}\right)^{2}
$$

which can be obtained by inserting (7) into (6). 
The melted phase can be distinguished from the crystalline one in a region increasing exponentially in size with decreasing temperature, or in the value $\delta-\delta_{\mathrm{c}}$, due to constant value of the dislocation core energy (of the order of $\varepsilon_{0} l_{0}$ ). This circumstance hampers the observation of the melted phase in small samples. In real experiments either the structure of the substrate or the coherence of an electron beam limit the visible region to within a few hundreds Angström.

Solitons also interact due to elastic strains in the bulk of the substrate. This interaction was first investigated by Gordon and Villain [7]. They found the interaction energy to be proportional to $l^{-2}$ with a coefficient independent of temperature. The exact calculation of the coefficient will be published elsewhere. Including this term in the energy density (1) we obtain a finite melting temperature even at $p=1,2$. However, the melting temperature may be rather small (proportional to the square root of the ratio of the elastic constants of the overlayer to the bulk). We expect it to be really small for overlayers of noble gases and not so small for overlayers of alkali atoms.

\section{References}

[1] Luther, A. and Pokrovsky, V., unpublished. See also : Luther, A., Timonen, J. and Pokrovsky, V., in Proceedings of the Erice Summer School on Phase Transition in Surface Films, Erice, Sicily, June 1979.

[2] Pokrovsky, V. L., Talapov, A. L., ZhETF 75 (1978) 1151. Sov. Phys. JETP 48 (1978) 379.

[3] Pokrovsky, V. L. and Talapov, A. L., Phys. Rev. Lett. 42 (1979) 65.

Pokrovsky, V. L.,'TALAPOv, A. L., ZhETF 78 (1980) 269. Sov. Phys. JETP 51 (1980) 134.

[4] Lyuksyutov, I. F., Pis'ma ZhETF 32 (1980) 593.

[5] Coppersmith, S. N., Fisher, D. S., Halperin, B. I., Lee, P. A. and Brinkman, W. F., Phys. Revi. Lett. 46 (1981) 549.

[6] Kosterlitz, J. M. and Thouless, D. J., J. Phys. C 6 (1973) 1181.

[7] Gordon, M. B., Villain, J., J. Phys. C 12 (1979) L-151. 